\title{
Successful Treatment of Refractory Cancer Pain and Depression with Continuous Intrathecal Administration of Dexmedetomidine and Morphine: A Case Report
}

Ge Huang · Guo Liu · Zhiguo Zhou · Jinfeng Yang $\cdot$ Chen Su (D)

Received: May 26, 2020 / Published online: July 12, 2020

(C) The Author(s) 2020

\section{ABSTRACT}

Patients who have refractory cancer pain suffer both physically and psychologically. Cancer pain management has improved over the past few decades. However, the treatment of refractory cancer pain is still challenging all over the world. Intraspinal analgesia has become an effective strategy to treat refractory pain in patients with cancer. In this report, we present a patient receiving a large dose of intrathecal opioids for refractory cancer pain, and who is also afflicted with pain-induced depression. Dexmedetomidine (DEX) was used as part of a multimodal analgesic regimen that successfully alleviated both the patient's pain and depression. An intrathecal infusion of DEX may serve as an adjuvant drug in the treatment of cancer pain and pain-related depression.

Digital Features To view digital features for this article go to https://doi.org/10.6084/m9.figshare.12582596.

G. Huang · G. Liu · Z. Zhou · J. Yang · C. Su ( $ه)$ Department of Anesthesiology and Pain Medicine, Hunan Cancer Hospital/The Affiliated Cancer Hospital of Xiangya School of Medicine, Changsha 410013, China

e-mail: suchen2007best@163.com

G. Huang

University of South China, Hengyang 421001,

Hunan, China
Keywords: Depression; Dexmedetomidine; Intrathecal analgesia; Refractory cancer pain

\section{Key Summary Points}

Why carry out this study?

A few studies reported that emotion disorder, like depression, is pretty common in patients with cancer pain, and this patient had depressive symptoms with unrelieved severe pain.

This case showed that coadministration of DEX and morphine intrathecally is an effective and safe way to treat refractory cancer pain in the patient.

Intrathecal administration of DEX has alleviated depressive behaviors of this patient and it therefore might have antidepressive properties.

What was learned from this study?

This case provides evidence that the multimodal pain management with DEX participation could be an effective and safe treatment strategy for refractory cancer pain and pain-related depression.

Refractory cancer pain and accompanying depression deserve more attention and intervention to deliver better management both physically and psychologically. 


\section{INTRODUCTION}

Pain is one of the most prevalent symptoms in patients with advanced cancer [1]. Cancer pain can be effectively treated in most patients by following the guidelines of the three-step analgesic ladder [2]. In spite of aggressive treatment, $10-15 \%$ of patients with cancer still suffer from refractory cancer pain [3]. Prolonged pain can significantly affect patients, not only in terms of physical suffering but also mental anguish, which can produce depression in up to $20 \%$ of patients with cancer [4]. Depression itself leads to additional emotional and cognitive deficits, further impairing the effective treatment of pain [5]. This is especially true in patients with refractory cancer pain. Unrelieved severe pain negatively affects patients' daily activities, selfcare ability, social interactions, and overall quality of life [2]. For these reasons, the effective treatment of severe cancer pain and accompanying depression is of critical importance.

Intrathecal therapy is often an effective option for the treatment of refractory pain. Intrathecal therapy can also provide improved analgesia with a reduced need for opioids, decreasing opioid-related side effects compared to other routes of delivery $[6,7]$. However, as the dose of intrathecal opioids increases, inevitable side effects, such as nausea, vomiting, urinary retention, and itching, may occur. Multimodal pain management has become a standard of care in perioperative analgesia. By combining analgesics that work via different mechanisms, pain control is enhanced and the need for opioids is reduced along with their adverse effects. Unfortunately, intrathecal therapy is often not considered early as part of a multimodal protocol for patients with cancer and significant pain.

Dexmedetomidine (DEX), which is eight times more specific for the $\alpha_{2}$ receptor than clonidine is, is a specific and highly effective $\alpha_{2}$ receptor agonist [8]. Intravenous administration of DEX before general anesthesia can induce perioperative sedation, analgesia with hemodynamic stability, and reduce the need for inhalational and intravenous anesthetics and opioids during surgery [9]. DEX inhibits neuronal discharge and provides opioid-sparing analgesic effects with minimal respiratory depression [10].

DEX has been reported to be safe to use intrathecally. Previously published meta-analysis and reviews have shown that intrathecal infusions of DEX can shorten the onset time of spinal anesthesic as well as enhance and prolong their sensory and motor effects, and increasing the time to the need for pain rescue after spinal anesthesia [11, 12]. Khosravi et al. demonstrated that intrathecal DEX produced better outcomes in terms of analgesia, sedation, and hemodynamic parameters compared to intravenous DEX [13]. Intrathecal infusions of DEX appear to activate $\alpha_{2 \mathrm{~A}}$ and $\alpha_{2 \mathrm{C}}$ receptors in the spinal cord and lead to a decrease in the release of substance $P$, thereby reducing pain [14]. Consequently, these facts support the use of intrathecal DEX in cancer pain treatment. In addition, studies have also shown that DEX has antidepressive effects in an animal model $[15,16]$. Overall, the mechanisms of antinociception and antidepression seen with DEX are not entirely understood.

In this report, we present a case of a 53-yearold female patient with refractory cancer pain receiving a large dose of intrathecal morphine, whose pain and pain-related depression were significantly improved with the addition of intrathecal DEX. Pain intensity was measured using Numeric Rating Scale score (NRS). The Hamilton Depression Rating Scale (HAMD-6) and the simplified EORTC QLQ-C30 metrics were used to evaluate patients' depression and quality of life, respectively [17].

\section{CASE REPORT}

This is a 53-year-old female patient with a 1 year and 5 months' history of primary right bronchial lung cancer with bilateral pleural and bone metastases. Her tumor was a poorly differentiated adenocarcinoma, peripheral type, with localized sarcoma-like changes. Genetic testing suggested the presence of epidermal growth factor receptor (EGFR) wild-type c-Met exon 14 skipping alterations. The patient provided her informed consent to publish the 
article and all procedures were conducted as part of standard care/treatment.

The patient first consulted the pain clinic in December of 2018, complaining of intermittent pain in the right chest. She was initially treated with hydrocodone hydrochloride $10 \mathrm{mg} / 12 \mathrm{~h}$ and aminophenol hydrocodone $5 \mathrm{mg} / 6 \mathrm{~h}$ orally. These medications reduced her pain and stabilized her NRS to a score of 3-4. Six months later, she began complaining of a significant persistent stinging pain in the left hip and thigh. A CT examination indicated that the left iliac bone had metastatic lesions. From June 2019 to December 2019, she was admitted several times for pain control. The dosage of oxycodone hydrochloride was gradually increased to $120 \mathrm{mg} / 12 \mathrm{~h}$ while the aminophenol oxycodone remained unchanged, as shown in Fig. 1. Unfortunately, she did not obtain adequate pain relief and complained of pain in her left hip and thigh, with an NRS of 5-6. During this period, the patient was being treated with immunotherapy (bozitinib $200 \mathrm{mg}$ bid) for primary lung cancer and experienced limited improvement.

The patient had persistent severe pain that was not relieved by large doses of opioids administrated orally. In addition to her physical condition, her psychological health was also worsening. According to the HAMD- 6 test, the patient was demonstrating restlessness, anxiety, sleep deprivation, and was in a state of panic since the beginning of December 2019. After consultation with the pain service, she was diagnosed with pain-related depression and was started on the antidepressant fluvoxamine maleate $100 \mathrm{mg}$ every night to treat her depressive symptoms. The patient was on the

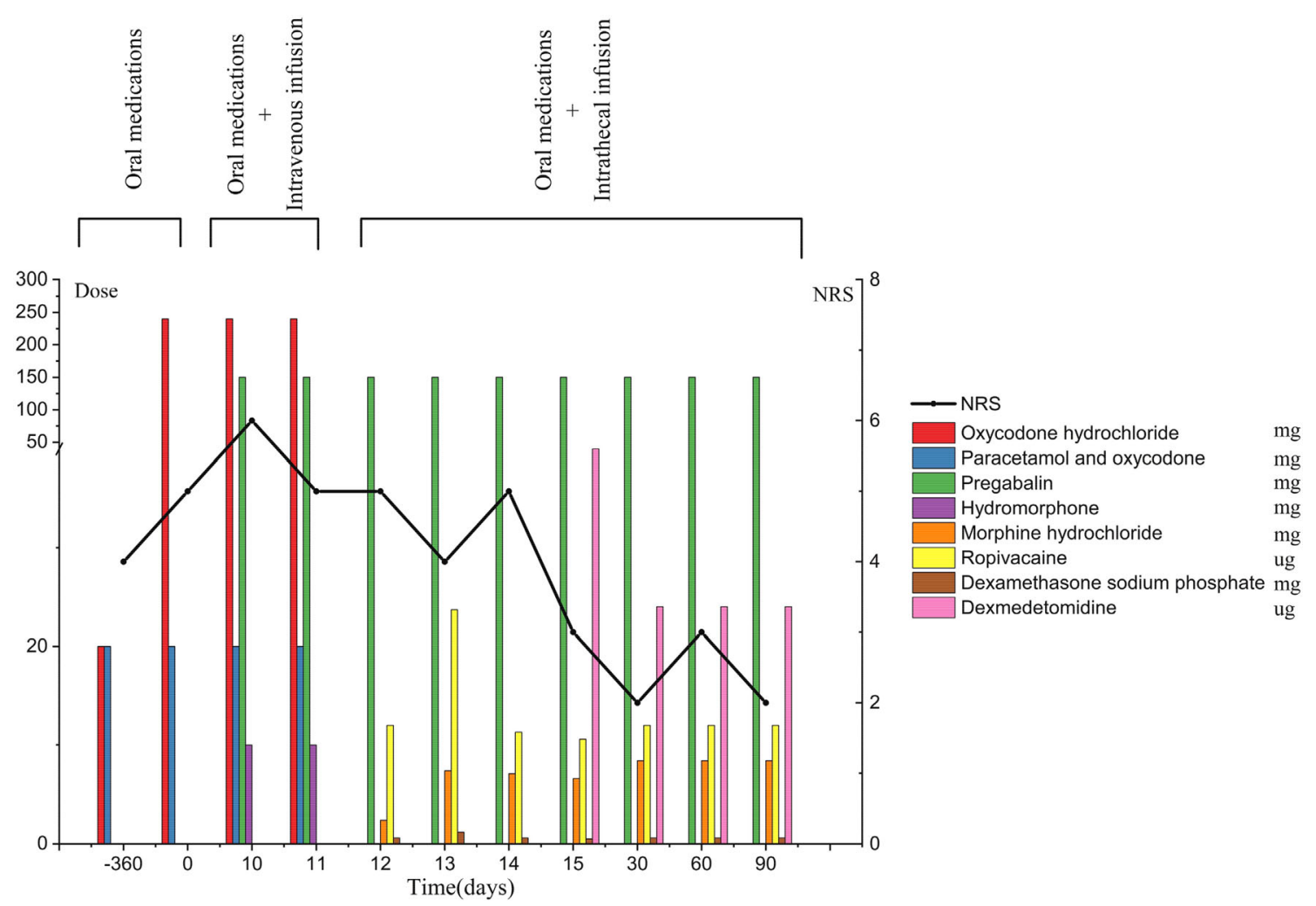

Fig. 1 Analgesia therapy was tracked for the patient from December 2018 to March 2020. There are three phases: oral medication only, oral medication combined with intravenous infusions, and oral medication combined with intrathecal infusions. December 1, 2019 is set as 0 on the abscissa. Medicine dosages are noted by color bars and the black line indicated the NRS scores 
antidepressant for 1 week, but failed to improve on this therapy and refused to take it further. In order to acutely improve her pain control, a hydromorphone patient-controlled intravenous analgesia (PCIA) pump was started on December 11,2019 with a dose of $0.2 \mathrm{mg} / \mathrm{h}$ and a bolus of $0.5 \mathrm{mg}$. In addition, a CT was performed that demonstrated an increasing right upper lung mass and increasing right pleural and left iliac metastasis. The oncology service added the programmed cell death protein 1 (PD1) inhibitor nivolumab $140 \mathrm{mg}$ to treat her primary disease on the basis of bozitinib. In spite of the increased therapy, there was no improvement in her overall level of pain, which was still reported as an NRS of 6 . At this point, the patient was suffering from the knowledge of her worsening disease and from increasing pain. She reported feeling helpless and that her situation was hopeless; she was considering stopping her treatments. MRI imaging of her spine showed normal anatomy, with no evidence of metastasis. Consequently, the pain service implanted an intrathecal analgesic drug infusion pump at L3-4 on December 13, 2019. The intrathecal pump had a volume of $100 \mathrm{ml}$, contained morphine hydrochloride $20 \mathrm{mg}$, ropivacaine $0.1 \mathrm{mg}$, and dexamethasone $5 \mathrm{mg}$, and delivered an infusion rate of $0.5 \mathrm{ml} / \mathrm{h}$. Her oral medication was changed to pregabalin $75 \mathrm{mg} / 12 \mathrm{~h}$. Even after these changes, it was observed that the patient's pain was still poorly controlled. An additional $10 \mathrm{mg}$ of morphine was added to the pump mixture the next day and the speed was increased to $1 \mathrm{ml} / \mathrm{h}$. On the third day after pump placement, the patient was still complaining of significant pain. The morphine content was increased again to $50 \mathrm{mg}$ and the pump rate was lowered to $0.5 \mathrm{ml} / \mathrm{h}$. None of these adjustments achieved significant pain control and the NRS pain scored remained 4-5.

The morphine pump content was raised to $70 \mathrm{mg}$, with the patient receiving $0.35 \mathrm{mg} / \mathrm{h}$, without an obvious increase in analgesic effect. On the fourth day of intrathecal therapy, the possibility of opioid-induced tolerance and concern about the increased risk of side effects from high doses of opioids caused the pain service to consider supplementing the intrathecal infusion with another agent. DEX was selected and was added to the pump infusion. The intrathecal pump now contained morphine $70 \mathrm{mg}$, ropivacaine $0.1 \mathrm{mg}$, dexamethasone $5 \mathrm{mg}$, and DEX $200 \mu \mathrm{g}$; the infusion rate was set at $0.5 \mathrm{ml} / \mathrm{h}$. With this mixture and infusion rate, the patient reported significant improvement in the following week. After the addition of DEX, the patient reported an NRS that was maintained at 2-3. Subsequently, the patient was discharged home with this mixture and infusion rate. No serious side effects were observed in the hospital and the patient received frequent follow-up phone calls (Table 1). No serious side effects such as respiratory depression, bradycardia, or hypotension were reported. Additionally, questionnaire surveys were conducted using HAMD-6 and quality of life measurement (EORTC QLQ-C30), as shown in Figs. 2 and 3, respectively. The results showed positive changes in depressive symptoms and quality of life over the next 3 months.

Table 1 Frequency score comparison of side effects before and after DEX

\begin{tabular}{lll}
\hline Symptoms & Frequency & \\
\cline { 2 - 3 } Before & After \\
\hline Constipation & 5 & 5 \\
Nausea & 5 & 4 \\
Vomiting & 5 & 3 \\
Difficult urination & 5 & 5 \\
Somnolence & 5 & 3 \\
Urinary retention & 1 & 1 \\
Pruritus & 2 & 1 \\
Hallucinations & 1 & 1 \\
Respiratory depression & 1 & 1 \\
Hypotension & 1 & 1 \\
Hypertension & 1 & 1 \\
Bradycardia & 1 & 1 \\
\hline Scores firom & & 5
\end{tabular}

Scores from 1 to 5 correspond to the following five frequency stages: never, seldom, sometimes, usually, and always 


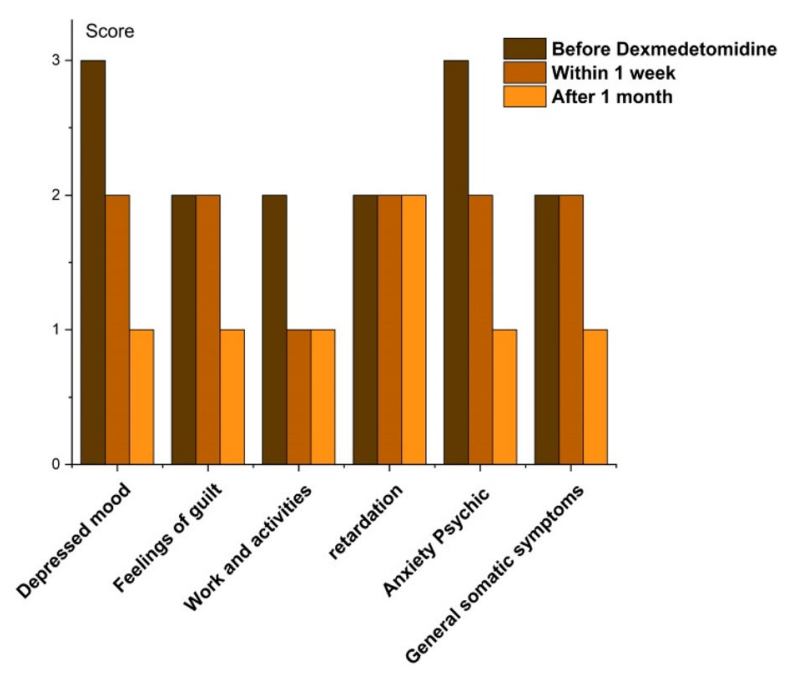

Fig. 2 HAMD-6: evaluation of the six item scales in three phases, without DEX infusion, within 1 week, and 1 month after DEX intrathecal treatment

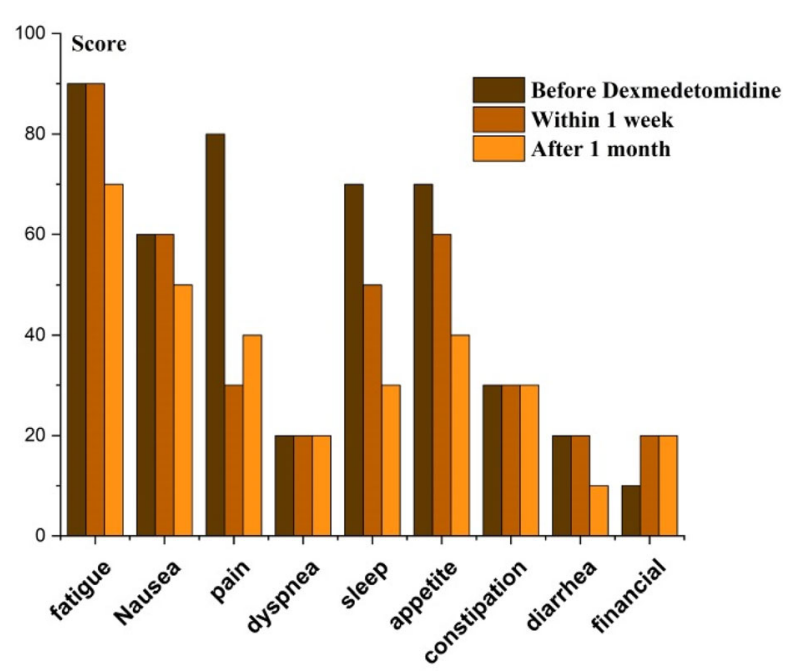

Fig. 3 EORTC QLQ-C30: evaluation of the three symptom scales and six single item scales in three phases

The patient, at the time of writing (May 2020), continues to receive intrathecal infusion, which is adjusted to her pain levels. She is currently receiving a $100 \mathrm{ml}$ mixture of morphine $90 \mathrm{mg}$, ropivacaine $0.1 \mathrm{mg}$, dexamethasone $5 \mathrm{mg}$, and DEX $200 \mu \mathrm{g}$. Her pain was controlled well at the pump rate of $0.5 \mathrm{ml} / \mathrm{h}$ and bolus of $1 \mathrm{ml}$.

\section{DISCUSSION}

This case demonstrates the treatment of refractory cancer pain by a continuous intrathecal infusion of DEX, morphine, and ropivacaine. DEX appears to be a safe and effective adjuvant therapy in the treatment of intractable cancer pain that is not responsive to traditional infusion combinations. Furthermore, coadministration of DEX with morphine intrathecally could reduce the overall opioid consumption required for acceptable pain control. As an added benefit, DEX also appeared to provide some relief of this patient's depression, although it is unclear if this was a direct or indirect effect. DEX is a widely used agent in anesthesia and has been reported to be effective when administered by both the intravenous and intrathecal routes $[9,13,18]$. Previous studies showed that systemically administrated DEX, in combination with morphine, could reduce opioid tolerance [19]. Additionally, many studies have shown that DEX reduces the consumption of opioids by $30-50 \%$, and synergistically enhances morphine analgesia [20, 21]. Roberts et al. reported that the combination of DEX and opioids could provide better analgesic effects than opioids alone, in the treatment of refractory cancer pain [22]. However, the analgesic mechanisms of DEX are not fully understood. There are highdensity $\alpha_{2}$ adrenergic receptors in both the spinal cord and at supraspinal levels [23]. The mechanism of antinociception is believed to be the result of stimulating $\alpha_{2}$ receptors in the central nervous system and spinal cord, thereby decreasing neuronal activity [10]. DEX was also found to have an effect on NR2B, a functional subunit of the $N$-methyl-D-aspartate (NMDA) receptor, phosphorylation in the spinal cord, and regulates protein kinase C (PKC) levels, thereby exerting an analgesic effect in an animal model of hyperalgesia [24].

In this case, tumor progression and unrelieved refractory cancer pain affected the patient's physical and psychological state, destroying her confidence in her overall treatment. As a result of worsening pain, this patient had increasing opioid consumption without adequate pain relief until DEX was added as an 
adjuvant. DEX, when it was given intrathecally, enhanced the analgesic effects of morphine and reduced the dosage of morphine required. This result is highly consistent with previous studies, showing that DEX leads to a reduction in the expression of morphine analgesic tolerance and enhances its analgesic effects [19].

The intrathecal administration of DEX is a viable treatment to significantly reduce the consumption of opioids and their side effects in patients with refractory cancer pain. We observed no side effects from the addition of DEX in this particular patient. These results are consistent with previous research demonstrating that patients did not report DEX-related side effects while receiving it intrathecally as an adjuvant pain therapy $[18,25]$. Table 1 records the overall side effects experienced by this patient. It is worth noting that side effects previously experienced by the patient, drowsiness, nausea, and vomiting, were reduced after DEX administration. Although the most common side effects of opioids (such as constipation and difficulty urinating) still existed, urine retention, hypotension, and bradycardia were not reported. Additionally, the patient did not experience serious adverse reactions during opioid administration such as respiratory depression, hypotension, bradycardia, and hypertension. We have demonstrated that an intrathecal injection of morphine combined with DEX can be a safe and effective method to treat refractory cancer pain in some patients, especially for those patients requiring a large amount of opioids.

Jack et al. reported that over one-third of patients with cancer experience depression, with the alleviation of pain leading to improved mood [26]. It is worth mentioning that this patient had symptoms of depression that improved after her pain improved. This improvement in depression could simply be the result of her improved pain control. However, in Figs. 2 and 3, as time progressed, it is notable that symptoms such as depression, feelings of guilt, quality of sleep, and appetite improved. This result may indicate that DEX could have some antidepressive properties.

Studies have shown that intrathecal injections of tramadol and DEX demonstrate better antidepressant effects than tramadol alone. When electroshock shock therapy is used to treat depression, the addition of DEX has been shown to improve the patient's learning and memory functions [27]. Further, it has been demonstrated that a reduction of $\alpha_{2}$ adrenergic receptors or a knockout of the $\alpha_{2}$ gene can cause an increase in depression-like or anxiety-like behaviors in rodents. This supports the concept that $\alpha_{2}$ adrenergic receptors may play an important role in emotional health [28]. Additionally, the intravenous administration of DEX has been shown to improve depressive behaviors in an animal model $[15,16]$. Consequently, DEX, as an $\alpha_{2}$ adrenergic receptor agonist, may have potential beneficial effects on mood. This makes DEX a suitable adjuvant analgesia medication for patients with cancer pain who have depressive symptoms.

\section{CONCLUSION}

This case demonstrates that a continuous intrathecal infusion of DEX and morphine is an effective and safe treatment option for patients who have refractory cancer pain and depressive symptoms. However, there is limited data evaluating the use of DEX in this particular scenario and controlled trials are required to further evaluate the efficacy and safety of DEX in this patient population. In particular, the potential mechanism of DEX's antidepressive effect, if direct, remains unclear. Our report could be a good start to inspire further research and more convincing clinical study in the future.

\section{ACKNOWLEDGEMENTS}

We thank all participants in the study.

Funding. No funding or sponsorship was received for this study or publication of this article. The Rapid Service Fee was funded by the authors.

Authorship. All named authors meet the International Committee of Medical Journal Editors (ICMJE) criteria for authorship for this 
article, take responsibility for the integrity of the work as a whole, and have given their approval for this version to be published.

Disclosure. Ge Huang, Guo Liu, Zhiguo Zhou, Jinfeng Yang, and Chen Su have nothing to disclose.

Compliance with Ethics Guidelines. The patient provided her informed consent to publish the article and all procedures were conducted as part of standard care/treatment.

Open Access. This article is licensed under a Creative Commons Attribution-NonCommercial 4.0 International License, which permits any non-commercial use, sharing, adaptation, distribution and reproduction in any medium or format, as long as you give appropriate credit to the original author(s) and the source, provide a link to the Creative Commons licence, and indicate if changes were made. The images or other third party material in this article are included in the article's Creative Commons licence, unless indicated otherwise in a credit line to the material. If material is not included in the article's Creative Commons licence and your intended use is not permitted by statutory regulation or exceeds the permitted use, you will need to obtain permission directly from the copyright holder. To view a copy of this licence, visit http:// creativecommons.org/licenses/by-nc/4.0/.

\section{REFERENCES}

1. Syrjala KL, et al. Psychological and behavioral approaches to cancer pain management. J Clin Oncol. 2014;32(16):1703-11.

2. Swarm RA, et al. Adult cancer pain, version 3.2019, NCCN clinical practice guidelines in oncology. J Natl Compr Cancer Netw. 2019;17(8):977-1007.

3. Gallagher R, Hawley P, Yeomans W. A survey of cancer pain management knowledge and attitudes of British Columbian physicians. Pain Res Manag. 2004;9(4):188-94.

4. Pitman A, et al. Depression and anxiety in patients with cancer. BMJ. 2018;361:k1415.
5. Baune BT, et al. The role of cognitive impairment in general functioning in major depression. Psychiatry Res. 2010;176(2-3):183-9.

6. Bhaskar AK. Interventional management of cancer pain. Curr Opin Support Palliat Care. 2012;6(1):1-9.

7. Thakur A, et al. Intrathecal clonidine as an adjuvant to hyperbaric bupivacaine in patients undergoing inguinal herniorrhaphy: a randomized doubleblinded study. J Anaesthesiol Clin Pharmacol. 2013;29(1):66-70.

8. Gertler R, et al. Dexmedetomidine: a novel sedative-analgesic agent. Proc (Bayl Univ Med Cent). 2001;14(1):13-21.

9. Khan ZP, et al. Effects of dexmedetomidine on isoflurane requirements in healthy volunteers. 1 : pharmacodynamic and pharmacokinetic interactions. Br J Anaesth. 1999;83(3):372-80.

10. Chan AK, Cheung CW, Chong YK. Alpha-2 agonists in acute pain management. Expert Opin Pharmacother. 2010;11(17):2849-68.

11. Shen $\mathrm{QH}$, et al. Dexmedetomidine as an adjuvant for single spinal anesthesia in patients undergoing cesarean section: a system review and meta-analysis. J Int Med Res. 2020;48(5):300060520913423.

12. Liu S, et al. Effect of 5- $\mu$ g dose of dexmedetomidine in combination with intrathecal bupivacaine on spinal anesthesia: a systematic review and metaanalysis. Clin Ther. 2020;42(4):676-676.

13. Khosravi F, Sadeghi N, Jarineshin H. The effect of dexmedetomidine on spinal anesthesia quality and hemodynamic changes in patients undergoing inguinal hernia repair surgery: intravenous versus intrathecal. Eur J Clin Pharmacol. 2020;76(7): 923-928.

14. Manne GR, Upadhyay MR, Swadia V. Effects of low dose dexmedetomidine infusion on haemodynamic stress response, sedation and post-operative analgesia requirement in patients undergoing laparoscopic cholecystectomy. Indian J Anaesth. 2014;58(6):726-31.

15. Stone EA, et al. Antidepressant-like action of intracerebral 6-fluoronorepinephrine, a selective full alpha-adrenoceptor agonist. Int J Neuropsychopharmacol. 2011;14(3):319-31.

16. Millan MJ. The role of monoamines in the actions of established and "novel" antidepressant agents: a critical review. Eur J Pharmacol. 2004;500(1-3): $371-84$

17. Aaronson NK, et al. The European Organization for Research and Treatment of Cancer QLQ-C30: a 
quality-of-life instrument for use in international clinical trials in oncology. J Natl Cancer Inst. 1993;85(5):365-76.

18. Paramasivan A, et al. Intrathecal dexmedetomidine and postoperative pain: a systematic review and meta-analysis of randomized controlled trials. Eur J Pain. 2020. https://doi.org/10.1002/ejp.1575.

19. Gursoy S, et al. Effects of alpha 2-adrenoceptor agonists dexmedetomidine and guanfacine on morphine analgesia and tolerance in rats. Upsala J Med Sci. 2011;116(4):238-46.

20. Sadhasivam S, Boat A, Mahmoud M. Comparison of patient-controlled analgesia with and without dexmedetomidine following spine surgery in children. J Clin Anesth. 2009;21(7):493-501.

21. Wallace S, Mecklenburg B, Hanling S. Profound reduction in sedation and analgesic requirements using extended dexmedetomidine infusions in a patient with an open abdomen. Mil Med. 2009;174(11):1228-30.

22. Roberts SB, et al. Dexmedetomidine as an adjuvant analgesic for intractable cancer pain. J Palliat Med. 2011;14(3):371-3.

23. Unnerstall JR, Kopajtic TA, Kuhar MJ. Distribution of alpha 2 agonist binding sites in the rat and human central nervous system: analysis of some functional, anatomic correlates of the pharmacologic effects of clonidine and related adrenergic agents. Brain Res. 1984;319(1):69-101.

24. Zheng Y, et al. Dexmedetomidine prevents remifentanil-induced postoperative hyperalgesia and decreases spinal tyrosine phosphorylation of N-methyl-d-aspartate receptor 2B subunit. Brain Res Bull. 2012;87(4-5):427-31.

25. Bi YH, et al. Effect of different doses of intrathecal dexmedetomidine as an adjuvant combined with hyperbaric ropivacaine in patients undergoing cesarean section. Front Pharmacol. 2020;11:342.

26. Jack L, et al. Pain and depression in cancer patients: a longitudinal study. BMJ Support Palliat Care 2011;1(Suppl 1):A11.

27. Gao X, et al. Dexmedetomidine protects against learning and memory impairments caused by electroconvulsive shock in depressed rats: involvement of the NMDA receptor subunit 2B (NR2B)-ERK signaling pathway. Psychiatry Res. 2016;243:446-52.

28. Schramm NL, McDonald MP, Limbird LE. The alpha(2a)-adrenergic receptor plays a protective role in mouse behavioral models of depression and anxiety. J Neurosci. 2001;21(13):4875-82. 